\title{
Casimir Forces and Boundary Conditions in One Dimension: Attraction, Repulsion, Planck Spectrum, and Entropy
}

\author{
Timothy H. Boyer \\ Department of Physics, City College of the City \\ University of New York, New York, New York 10031
}

(Dated: October 24, 2002)

\begin{abstract}
Quantities associated with Casimir forces are calculated in a model wave system of one spatial dimension where the physical ideas are transparent and the calculations allow easy numerical evaluation. The calculations show strong dependence upon fixed- or free-end (Dirichlet or Neumann) boundary conditions for waves on a one-dimensional string, analogous to infinitely-conducting or infinitely-permeable materials for electromagnetic waves. 1) Due to zero-point fluctuations, a partition in a one-dimensional box is found to be attracted to the walls if the wave boundary conditions are alike for the partition and the walls, but is repelled if the conditions are different. 2) The use of Casimir energies in the presence of zero-point radiation introduces a natural maximum-entropy principle which is satisfied only by the Planck spectrum for both like and unlike boundary conditions between the box and partition. 3)The one-dimensional Casimir forces increase or decrease with increasing temperature depending upon like or unlike boundary conditions. The Casimir forces are attractive and increasing with temperature for like boundary conditions, but are repusive and decreasing with temperature for unlike boundary conditions. 4) In the high-temperature limit, there is a temperature-independent Casimir entropy for like boundary conditions, but a vanishing Casimir entropy for unlike boundary conditions. These one-dimensional results have counterparts for electromagnetic Casimir forces in three dimensions.
\end{abstract}




\section{INTRODUCTION}

There has been an ever-increasing interest in Casimir forces during the past halfcentury.[1] What began as an obscure phenomenon in electrodynamics[2] has became of general theoretical interest in quantum field theory[3], has been connected to wave phenomena in acoustics[4] and boating,[5] and is now of technological concern in connection with microelectromechanical devices.[6] In particular, there have been accurate experimental measurements of electromagnetic Casimir forces[7] and suggestions that such forces will create a "stiction" which will bind surfaces together at small separations. In order to overcome such stiction, the repulsive aspects of Casimir forces have recently been reinvestigated.[8] In this article we review some aspects of thermodynamic Casimir forces and energies in a simple model system allowing extraction of the essential physics with a minimum of mathematical complexity.

Our work presents simple examples of physical aspects of Casimir forces in one spatial dimension. We begin with an example of positive and negative Casimir energies at zero-temperature in connection with changes in wave boundary conditions. Next we give examples of the natural maximum-entropy principle for thermal radiation leading to the Planck spectrum, noting that the result indeed holds for the repulsive case, as was shown earlier for the attractive case. Then we give examples of the attractive and repulsive Casimir forces due to thermal radiation at finite temperature. In one spatial dimension, we find that whereas the attractive force increases with increasing temperature, the repulsive force diminishes to zero with increasing temperature. Finally, we consider the entropy changes of the wave system and note the temperature-independent Casimir entropy which arises in the attractive but not the repulsive case at high temperature. Some of these aspects of Casimir forces already appear in the literature for more complicated systems demanding more elaborate mathematical calculation. The examples illustrate the variety of behaviors encountered for different boundary conditions.

\section{CASIMIR FORCES AND BOUNDARY CONDITIONS}

Casimir forces are those arising from the discrete normal mode structure of a wave system. Thus if we consider random radiation in a box, the introduction of a partition will change the normal mode structure of the box and so lead to changes in forces and energies. The changes of these forces and energies associated with the placement of the partition are termed Casimir energies. The nature of these Casimir forces and energies will depend upon the energy spectrum of the waves, upon the number of spatial dimensions, and upon the boundary conditions at the partition and walls of the box.

In this article we will consider thermodynamic Casimir forces in one space dimension. Thus we may imagine waves on a string between two supports, or electromagnetic waves all of whose wave vectors point in a single direction perpendicular to the cavity walls. Furthermore, the energy spectrum of the radiation is not taken arbitrarily[9] but rather is assumed to correspond to thermodynamic equilibrium and so to satisfy the Wien displacement the-

orem. Accordingly, the average energy $\mathcal{U}(\omega, T)$ per normal mode is related to the angular frequency $\omega$ and temperature $T$ by[10]

$$
\mathcal{U}(\omega, T)=-\omega \phi^{\prime}(\omega / T)
$$


where the thermodynamic potential[11] $\phi(\omega / T)$ is a function of the single variable $\omega / T$. Of course, the analysis given here can be extended easily to more general energy spectra.

Wien's displacement theorem for thermal radiation allows two extremes where the energy $\mathcal{U}$ per normal mode is independent of one of its two variables. If the derivative of the thermodynamic potential is a constant $\phi^{\prime}(\omega / T)=-$ const, then $\mathcal{U}(\omega, T)=$ const $\times \omega$ corresponding to the familiar zero-point energy where the constant is chosen as related to Planck's constant,

$$
\mathcal{U}=(1 / 2) \hbar \omega
$$

On the other hand, if the derivative of the thermodynamic potential is equal to the inverse of its argument $\phi^{\prime}(\omega / T)=-$ const $\times(T / \omega)$, then we find the familiar energy equipartition result $\mathcal{U}=$ const $\times T$ where the constant is chosen as Boltzmann's constant,

$$
\mathcal{U}=k_{B} T
$$

\section{A. Casimir Energy for Zero-Point Radiation}

At zero temperature $T=0$, a one-dimensional box of length $L$ contains the zero-point energy

$$
U_{z p}(L)=\Sigma_{n}(1 / 2) \hbar \omega_{n}
$$

where the sum is over all normal modes of wave oscillation. If a partition is introduced at $x$ into the box of length $L$, then the Casimir energy is the change of energy $\Delta U_{z p}(x, L)$ when the partition is at distance $x$ from one of the ends compared to when it is in the middle of the box is

$$
\Delta U_{z p}(x, L)=U_{z p}(x)+U_{z p}(L-x)-\left[U_{z p}(L / 2)+U_{z p}(L / 2)\right]
$$

where we have summed over all the energies on both sides of the partition for the two cases. Realistic systems will not enforce the boundary conditions at very small wavelengths so that these sums should actually involve only a finite number of waves. It is natural to imagine a smooth cut-off function $F$ dependent upon wavelength $\Lambda$ of the form $F(\Lambda \omega / c)$ where $c$ is the wave speed. One example is $F(\Lambda \omega / c)=\exp (-\Lambda \omega / c)$. The energy sums in (5) are evaluated for a finite cut-off $\Lambda$, and then the cut-off is taken to zero to remove the dependence upon the cut-off. It turns out that the energy found in this limit is independent of the the choice of the smooth cut-off function $F$.[12] Provided that the boundary conditions are enforced down to some wavelength $\Lambda$ smaller than any of the dimensions of the system, the cut-off result for finite $\Lambda$ will agree with the no-cut-off limit $\Lambda=0$.

For Casimir forces in nature due to electromagnetic fields where we wish to avoid the details of the interaction with the walls, it is natural to choose either perfectly-conducting boundary conditions or else infinitely-permeable boundary conditions.[13] In one spatial dimension, these correspond to Dirichlet or Neumann boundary conditions analogous to a fixed or free end of a string.[14] If we imagine the partition to enforce the same boundary conditions as the walls (e.g., both partition and walls are good conductors $\cong$ fixed ends), then the normal modes on a string of length $L$ will have wave numbers $k_{n}=n \pi / L, n=1,2,3, \ldots$. On the other hand, if the partition enforces the opposite boundary conditions from the walls (e.g., a highly permeable partition and good conducting walls $\cong$ free end and fixed end), then the normal modes on a string of length $L$ have wave numbers $k_{n}=(n-1 / 2) \pi / L$, $n=1,2,3, \ldots$ We can discuss both cases at once by writing $k_{n}=(n-\alpha / 2) \pi / L$ where 
$\alpha=0$ for like boundary conditions for partition and walls, and $\alpha=1$ for unlike boundary conditions.

The Casimir energy at zero-temperature can be calculated easily by using an exponential cut-off function and summing the geometric series,

$$
\begin{gathered}
\Delta U_{z p}(x, L)=\lim _{\Lambda \rightarrow 0}\left\{\sum_{n=1}^{\infty} \frac{\hbar}{2} \frac{c \pi(n-a / 2)}{x} \exp \left(-\Lambda \frac{\pi(n-a / 2)}{x}\right)+\right. \\
\left.+\sum_{n=1}^{\infty} \frac{\hbar}{2} \frac{c \pi(n-a / 2)}{L-x} \exp \left(-\Lambda \frac{\pi(n-a / 2)}{L-x}\right)-2 \sum_{n=1}^{\infty} \frac{\hbar}{2} \frac{c \pi(n-a / 2)}{L / 2} \exp \left(-\Lambda \frac{\pi(n-a / 2)}{L / 2}\right)\right\} \\
=\lim _{\Lambda \rightarrow 0}\left\{-\frac{\hbar c}{2} \frac{\partial}{\partial \Lambda}\left[\frac{\exp \left(\frac{\Lambda \pi(1-a / 2)}{x}\right)}{1-\exp \left(-\frac{\Lambda \pi}{x}\right)}+\frac{\exp \left(\frac{\Lambda \pi(1-a / 2)}{L-x}\right)}{1-\exp \left(-\frac{\Lambda \pi}{L-x}\right)}-2 \frac{\left.\exp \left(\frac{\Lambda \pi(1-a / 2)}{L / 2}\right)\right]}{1-\exp \left(-\frac{\Lambda \pi}{L / 2}\right)}\right]\right\} \\
= \\
\lim _{\Lambda \rightarrow 0}\left\{\left[\frac{\hbar c x}{\Lambda^{2} \pi}-\left(\frac{1}{24}-\frac{\alpha}{8}+\frac{\alpha^{2}}{16}\right) \frac{c \pi}{x}+\bigcirc(\Lambda)\right]+\right. \\
+\left[\frac{\hbar c(L-x)}{2 \Lambda^{2} \pi}-\left(\frac{1}{24}-\frac{\alpha}{8}+\frac{\alpha^{2}}{16}\right) \frac{c \pi}{(L-x)}+\bigcirc(\Lambda)\right] \\
\left.-2\left[\frac{\hbar c(L / 2)}{2 \Lambda^{2} \pi}-\left(\frac{1}{24}-\frac{\alpha}{8}+\frac{\alpha^{2}}{16}\right) \frac{c \pi}{(L / 2)}+\bigcirc(\Lambda)\right]\right\} \\
=-\pi \hbar c\left(\frac{1}{24}-\frac{\alpha}{8}+\frac{\alpha^{2}}{16}\right)\left(\frac{1}{x}+\frac{1}{L-x}-\frac{2}{L / 2}\right)
\end{gathered}
$$

Thus for partition and walls which enforce like boundary conditions (either both Dirichlet or both Neumann), the parameter $\alpha$ takes the value $\alpha=0$, and the Casimir energy at zero temperature is negative

$$
\Delta U_{z p}(x, L)=-\frac{\pi \hbar c}{24}\left(\frac{1}{x}+\frac{1}{L-x}-\frac{2}{L / 2}\right)
$$

giving an attractive force. On the other hand, for partition and walls which enforce unlike boundary conditions, one Dirichlet and one Neumann, $\alpha=1$ and the Casimir energy is positive

$$
\Delta U_{z p}(x, L)=\frac{\pi \hbar c}{48}\left(\frac{1}{x}+\frac{1}{L-x}-\frac{2}{L / 2}\right)
$$

giving a repulsive force.

Exactly the analogous situation is found for the electromagnetic Casimir forces between two parallel conducting plates or between two permeable plates, or between a conducting plate and a permeable plate. The original 1948 calculation by Casimir[2] involved two conducting plates, and the result has been recalculated from many points of view.[1] The force between a conducting plate and a permeable plate was given[13] in 1974 and has been recalculated several times recently.[15] 


\section{B. CASIMIR ENERGY FOR THE RAYLEIGH-JEANS SPECTRUM}

The zero-point-energy limit of Wien's law leads to the Casimir energies given in (7) and (8). It is also of interest to obtain the Casimir energies for the energy-equipartition limit of Wien's law. This corresponds to the Rayleigh-Jeans spectrum of radiation. The Casimir energies again take the form given in (5), but this time with the energy per normal mode in (3). Once again the calculation can easily be carried through analytically for both like and unlike boundary conditions,

$$
\begin{gathered}
\Delta U_{R J}(x, L, T)= \\
=\lim _{\Lambda \rightarrow 0}\left\{\sum_{n=1}^{\infty} k_{B} T \exp \left(-\Lambda \frac{\pi(n-\alpha / 2)}{x}\right)+\sum_{n=1}^{\infty} k_{B} T \exp \left(-\Lambda \frac{\pi(n-\alpha / 2)}{L-x}\right)+\right. \\
\left.-2 \sum_{n=1}^{\infty} k_{B} T \exp \left(-\Lambda \frac{\pi(n-\alpha / 2)}{L / 2}\right)\right\} \\
=\lim _{\Lambda \rightarrow 0}\left\{\frac{k_{B} T \exp \left(\frac{-\Lambda \pi(1-\alpha / 2)}{x}\right)}{1-\exp \left(\frac{-\Lambda \pi}{x}\right)}+\frac{k_{B} T \exp \left(\frac{-\Lambda \pi(1-\alpha / 2)}{L-x}\right)}{1-\exp \left(\frac{-\Lambda \pi}{L-x}\right)}-2 \frac{k_{B} T \exp \left(\frac{-\Lambda \pi(1-\alpha / 2)}{L / 2}\right)}{1-\exp \left(\frac{-\Lambda \pi}{L / 2}\right)}\right\} \\
\left.-2 k_{B} T\left[\frac{L / 2}{\Lambda \pi}+\frac{1}{2}(\alpha-1)+\frac{\pi \Lambda}{(L / 2)} C-\ldots\right]\right\}=0
\end{gathered}
$$

In both cases, like or unlike boundary conditions, the Rayleigh-Jeans spectrum gives no change in the Casimir energy. Indeed, the equipartition Rayleigh-Jeans spectrum is the unique spectrum which produces no Casimir energy changes associated with the placement of the Casimir partition, $\Delta U_{R J}(x, L, T)=0$, no matter whether the partition enforces the same boundary conditions as the walls or the opposite boundary conditions.

The vanishing changes in Casimir energies for the Rayleigh-Jeans spectrum found here in (9) agrees with the work of Revzen, Opher, Opher, and Mann[16] for the case of two parallel conducting plates and with the work of da Silva, Matos Neto, Placido, Revzen, and Santana[17] for the case of two parallel plates, one of which is conducting and one of which is permeable.

\section{NATURAL MAXIMUM ENTROPY PRINCIPLE FOR THERMAL RADIA- TION}

It has been pointed out recently that Casimir forces and zero-point radiation allow the derivation of the Planck spectrum as the unique radiation spectrum giving minimum Casimir energy changes over an ensemble of containers with partitions spaced uniformly across the volume.[18] The result has been submitted for publication in the case of one-dimensional Casimir energies and has been suggested in the electromagnetic case for a three-dimensional box, both cases assuming Dirichlet boundary conditions. The same energies are involved for Neumann boundary conditions. Here we note that the result holds also for mixed boundary conditions in the case of one-dimensional waves. 
We consider an ensemble of one-dimensional boxes of length $L$ with partitions placed at different locations $x$ in the boxes so as to give uniform spacing across the entire ensemble. If each of these boxes contains radiation at thermal equilibrium at the same temperature $T$, then the boxes will still have different average energies corresponding to different Casimir energies relating to the differing placements of their partitions. Just as it is natural to suggest that maximum particle entropy involves a uniform distribution of particles across the volume of a box, it is natural to suggest that maximum radiation entropy involves the least possible variation in Casimir energies across the ensemble of boxes. Thus we are looking for that spectrum of random radiation which satisfies the Wien displacement theorem (and therefore has energy per normal mode $k_{B} T$ per normal mode at low frequency and $(1 / 2) \hbar \omega$ at high frequency) which gives a minimum for the sum of the absolute values of the Casimir energies taken across the ensemble of boxes,

$$
I=\Sigma_{i}\left|\Delta U\left(x_{i}, L, T\right)\right|
$$

This condition can be converted to the minimum for an integral

$$
I=\int_{x=\delta}^{x=L / 2} d x|\Delta U(x, L, T)|
$$

where $\delta$ is a fixed small cut-off distance. The fixed distance $\delta$ is chosen much smaller than any distance of interest in the situation; it is required by the divergence of the Casimir energy at small separations.

The integrand in (11) can be evaluated numerically for various suggested radiation spectra which provide smooth interpolations between $k_{B} T$ at low frequency and $(1 / 2) \hbar \omega$ at high frequency. Indeed one can assume various functional forms and then vary the parameters to provide the smallest value for the integral (11). For example, if one introduces parameters $C_{1}$ and $C_{2}$ into the functional form

$$
\mathcal{U}_{C_{1} C_{2}}(\omega, T)=\frac{C_{1} \omega \exp \left[-C_{2}(\omega / T)\right]}{1-\exp \left[-C_{1}(\omega / T)\right]}+(1 / 2) \omega
$$

then this provides a smooth interpolation of the required form for every positive value of $C_{1}$ and $C_{2}$. By numerical calculation for both like and unlike boundary conditions, it is found that the values of $C_{1}$ and $C_{2}$ which provide the smallest integral $I$ in (11) are $C_{1}=1$ and $C_{2}=1$, corresponding to exactly the Planck spectrum with zero-point energy

$$
\mathcal{U}_{P z p}(\omega, T)=\frac{\omega \exp [-(\omega / T)]}{1-\exp [-(\omega / T)]}+\frac{1}{2} \omega=\frac{\omega}{\exp (\omega / T)-1}+\frac{1}{2} \omega=\frac{1}{2} \omega \operatorname{coth}\left(\frac{1}{2} \frac{\omega}{T}\right)
$$

where for the numerical evaluation we have set $\hbar / k_{B}=1$. The case of like boundary conditions where the normal modes of the wave field are $k_{n}=n \pi / L, n=1,2, \ldots$ was calculated earlier and submitted for publication.[18] The case of unlike boundary conditions where the normal modes of the field are $k_{n}=(n-1 / 2) \pi / L$ has now been completed and is found by numerical calculation to lead to the Planck law as the spectrum providing a minimum for the integral $I$ in (11). For this case and for all spectra which have been tested, the Planck spectrum with zero-point radiation gave a smaller integral in (11).

An idea of what is involved can be seen in the Figs 1, 2, and 3. In Fig. 1, we plot the energy per normal mode $\mathcal{U}$ versus frequency $\omega$ for zero-point radiation, for the Rayleigh-Jeans 
spectrum, and for the Planck spectrum with zero-point radiation at two different temperatures. We note how smoothly the Planck spectrum interpolates between the equipartition behavior at low frequency and zero-point energy at high frequency. Figures 2 and 3 give the Casimir energies in a one-dimensional box with a partition at location $x$ for these same radiation spectra. In Figure 2 (like boundary conditions), the partition has the same boundary conditions as the walls of the box, while in Figure 3 (unlike boundary conditions), the partition has opposite boundary conditions from the walls. Thus we see the negative zero-point Casimir energy (7) in Fig. 2 and the positive zero-point Casimir energy (8) in Figure 3. The Rayleigh-Jeans spectrum gives zero Casimir energies (9) for both cases. The Planck spectrum with zero-point energy provides a smooth interpolating Casimir energy between these two extremes. One should notice how the Planck spectrum with zero-point energy hugs the axis of both the figures 3 and 4 before leaving the axis to join the zero-point curve at small separations $x$. This hugging-the-axis corresponds to small Casimir energies and accounts for the minimum behavior of the test integral in (11). It is easy to graph the Casimir energies for other interpolating spectra. One finds that other interpolating spectra satisfying the Wien displacement law do not hug the axis nearly so closely and therefore do not give a minimum for the test integral (11).[19]

We note that in both these cases, the presence of zero-point radiation is absolutely necessary. In the absence of zero-point radiation, there would be no non-zero minimum for the integral $I$; rather the energy would diverge to the Rayleigh-Jeans spectrum where the Casimir energies vanish for both like and unlike boundary conditions. It is precisely the presence of zero-point radiation which prevents the familiar "ultraviolet catastrophe" for thermal radiation.

\section{CASIMIR FORCES AT FINITE TEMPERATURE}

At zero temperature, the Casimir forces $X_{z p}$ on a partition can be obtained by using the Casimir energies in (7) and (8) as potential functions

$$
\Delta X_{z p}=-d\left(\Delta U_{z p}\right) / d x
$$

Accordingly, we find the attractive force

$$
\Delta X_{z p}=-\frac{\pi \hbar c}{24}\left(\frac{1}{x^{2}}-\frac{1}{(L-x)^{2}}\right)
$$

for the case of like boundary conditions $(\alpha=0)$, and the repulsive force

$$
\Delta X_{z p}=\frac{\pi \hbar c}{48}\left(\frac{1}{x^{2}}-\frac{1}{(L-x)^{2}}\right)
$$

for the case of unlike boundary conditions $(\alpha=1)$.

At finite temperature $T$, it is the derivative of the change in the Helmholtz free energy $\Delta F$ which gives the force on the partition. The Helmholtz free energy $\mathcal{F}(\omega, T)$ per wave normal mode in the case of the Planck spectrum with zero-point radiation is given by

$$
\mathcal{F}_{P z p}(\omega, T)=k_{B} T \ln \left[2 \sinh \left(\frac{\hbar \omega}{2 k_{B} T}\right)\right]=\frac{1}{2} \hbar \omega+k_{B} T \ln \left[1-\exp \left(\frac{-\hbar \omega}{k_{B} T}\right)\right]
$$


The Helmholtz free energy in a box is found by summing the Helmholtz free energies of the radiation modes

$$
F(L, T)=\Sigma_{n} \mathcal{F}\left(\omega_{n}, T\right)
$$

and the change in the Helmholtz free energy due to a change in the position of a partition in the box is

$$
\Delta F(x, L, T)=F(x, T)+F(L-x, T)-2 F(L / 2, T)
$$

Because of the zero-point energy, the change in Helmholtz free energy for the Planck spectrum with zero-point radiation requires the use of a temporary cut off as for our calculation in Eq. (6). For numerical evaluation, one may separate out the divergent zero-point energy contribution $\Delta F_{z p}$ and deal with the convergent thermal part $\Delta F_{P T}=\Delta F_{P z p}-\Delta F_{z p}$. The zero-point Helmholtz free energy $\Delta F_{z p}$ is identical to the zero-point energy $\Delta U_{z p}$ given in (6).

However, rather than dealing with the total Helmholtz free energy $\Delta F(x, L, T)$ as a potential function for forces, it seems easier to sum the forces due to the individual normal modes. The force $\mathcal{X}(\omega, L, T)$ on the boundary due to a single normal mode in a box is given by the derivative of the Helmholtz free energy of the normal mode in (17)

$$
\mathcal{X}_{P z p}(\omega, L, T)=-\left(\frac{\partial \mathcal{F}_{P z p}(\omega, T)}{\partial \omega}\right)_{T} \frac{d \omega}{d L}=\frac{\hbar \omega}{2 L} \operatorname{coth}\left(\frac{\hbar \omega}{2 k_{B} T}\right)
$$

Then the force $\Delta X$ on a partition at $x$ due to all the modes is

$$
\Delta X(x, L, T)=\sum_{n=1}^{\infty}\left[\mathcal{X}\left(\omega_{n}, x, T\right)-\mathcal{X}\left(\omega_{n}, L-x, T\right)\right]
$$

where once again we can include both sets of boundary conditions by including the parameter $\alpha$ in the normal modes in (21). For the Planck spectrum with zero-point radiation used in (20), this sum can be easily evaluated in closed form for the limits of zero-point radiation (which gives just $\Delta X_{P z p}$ above in (15) and (16)) or for the high-temperature limit of the Rayleigh-Jeans spectrum. For finite temperatures, the force can easily be evaluated numerically; we separate off the zero-point contribution and are left with a convergent sum in (21).

In the case of the high-temperature equipartition limit corresponding to the RayleighJeans law, the force in (20) due to a single normal mode becomes independent of $\omega$,

$$
\mathcal{X}_{R J}(\omega, L, T)=\frac{k_{B} T}{L}
$$

Therefore the sum over all the normal modes in (21) now involves the difference of two divergent sums. Accordingly, we temporarily introduce a smooth frequency-dependent cutoff function dependent upon the cut-off parameter $\Lambda$, and take the net force as the result when $\Lambda \rightarrow 0$,

$$
\begin{aligned}
\Delta X_{R J}(x, L, T) & =\lim _{\Lambda \rightarrow 0}\left\{\sum_{n=1}^{\infty} \frac{k_{B} T}{x} \exp \left(-\Lambda \frac{c \pi(n-\alpha / 2)}{x}\right)+\right. \\
- & \left.\sum_{n=1}^{\infty} \frac{k_{B} T}{L-x} \exp \left(-\Lambda \frac{c \pi(n-\alpha / 2)}{L-x}\right)\right\}
\end{aligned}
$$


Once again we are dealing with a geometric series which can be summed easily to give

$$
\begin{gathered}
\Delta X_{R J}(x, L, T)=\lim _{\Lambda \rightarrow 0}\left\{\frac{k_{B} T}{x}\left[\frac{x}{\Lambda \pi}+\frac{1}{2}(\alpha-1)+\frac{\Lambda \pi}{x} C+\ldots\right]+\right. \\
\left.-\frac{k_{B} T}{L-x}\left[\frac{L-x}{\Lambda \pi}+\frac{1}{2}(\alpha-1)+\frac{\Lambda \pi}{L-x} C+\ldots\right]\right\} \\
=\frac{k_{B} T}{2}(\alpha-1)\left(\frac{1}{x}-\frac{1}{L-x}\right)
\end{gathered}
$$

Thus at high temperatures for like boundary conditions where $\alpha=0$, there is a force attracting a partition to one of the walls

$$
\Delta X_{R J}(x, L, T)=-\frac{k_{B} T}{2}\left(\frac{1}{x}-\frac{1}{L-x}\right)
$$

We notice that this force, which arises from the discrete classical normal mode structure of the box, involves a different power of length from the zero-point force in (15), but both are significant only if $x$ or $L-x$ is small. On the other hand, in one dimension for unlike boundary conditions where $\alpha=1$, the net force on the partition in (24) vanishes entirely $\Delta X_{R J}(x, L, T)=0$ in the high-temperature Rayleigh-Jeans limit.

The results for the Casimir forces on a partition in one space dimension are shown in Figures 4 and 5 for like and unlike boundary conditions respectively. In the case of like boundary conditions shown in Fig. 4, the Casimir force is attractive at all separations and increases steadily as the temperature increases. In the case of unlike boundary conditions shown in Fig. 5, the Casimir force is repulsive at low temperatures for fixed displacement $x$, but then goes to zero as the temperature increases. In the limit of high temperature, both force curves go over to the results given by the Rayleigh-Jeans limit.

We should emphasize that the appearance of attractive or repulsive Casimir forces at zero temperature is not intuitively clear; changes in boundary conditions or shape or spatial dimension all seem involved.[1] Also, there seems to be some disagreement regarding the detailed temperature dependence of Casimir forces for like boundary conditions, though only increasingly attractive forces with increasing temperature seem to be expected, just as seen in Fig. 4.[20] In the case of repulsive forces involving mixed boundary conditions, the one-dimensional result in Fig. 5 involving diminishing repulsion with increasing temperature agrees with the behavior found by Ferrer and Grifols[21] for the repulsive Casimir-Polder force involving the electric polarizability of one particle and the magnetic polarizability of another.[22] However, it is qualitatively different from the increasing repulsion with temperature found by da Silva, Matos Neto, Placido, Revzen, and Santana for the hightemperature limit of two parallel plates one of which is a conductor and the other of which is highly permeable.[17]

\section{CASIMIR ENTROPY}

In addition to the surprising sign reversals found for Casimir forces and energies, and to the natural connection of Casimir energies with the Planck spectrum, there is an entropy aspect of the Casimir forces which seems curious. The Casimir entropy is the change 
in entropy of a wave system depending upon the placement of the system partition. The entropy $S(L, T)$ of radiation in a box is the sum over modes for the entropy $\mathcal{S}(\omega / T)$ of a normal mode which follows from the thermodynamic potential $\phi(\omega / T)$ as

$$
\mathcal{S}(\omega / T)=\phi(\omega / T)-\frac{\omega}{T} \phi^{\prime}(\omega / T) \quad \text { and } \quad S(L, T)=\sum_{n=1}^{\infty} \mathcal{S}\left(\omega_{n} / T\right)
$$

Accordingly, the Casimir entropy change $\Delta S(x, L, T)$ in a box of length $L$ at temperature $T$ due to a partition at $x$, is given by

$$
\Delta S(x, L, T)=S(x, T)+S(L-x, T)-2 S(L / 2, T)
$$

For the Planck spectrum

$$
\mathcal{S}_{P}\left(\frac{\omega}{T}\right)=-\ln \left[2 \sinh \left(\frac{\hbar \omega}{2 k_{B} T}\right)\right]+\frac{\hbar \omega}{2 k_{B} T} \operatorname{coth}\left(\frac{\hbar \omega}{2 k_{B} T}\right)
$$

the entropy changes for the case of like boundary conditions are given in Fig. 6, and those for unlike boundary conditions are given in Fig. 7. It is curious that in the case of like boundary conditions shown in Fig. 6, there is clearly a non-zero, temperature-independent limit for the Casimir entropy $\Delta S(x, L, T)$ at high temperature, whereas in the case of unlike boundary conditons shown in Fig. 7 there is not.

\section{A. ENTROPY CHANGES IN THE HIGH-TEMPERATURE LIMIT}

For fixed frequency $\omega$ and increasing temperature $T$, the Planck spectrum with zeropoint radiation goes over to the Rayleigh-Jeans spectrum. Similarly, for fixed length $x$ and increasing temperature $T$ the Casimir energy $\Delta U_{P z p}(x, L, T)$ for the Planck spectrum with zero-point radiation goes over to the Rayleigh-Jeans result $\Delta U_{R J}(x, L, T)=0$. However, the net force $\Delta X_{P z p}(x, L, T)$ and the net change in entropy $\Delta S_{P}(x, L, T)$ do not necessarily go to zero with increasing temperature. Indeed, these quantities go over to the values for the Rayleigh-Jeans spectrum, which values need not vanish. Thus at high temperature, Casimir forces are associated with changes in system entropy, not the vanishing changes in system energy.

Applying thermodynamic analysis to a partitioned one-dimensional box of radiation with a Rayleigh-Jeans spectrum at temperature $T$, an isothermal change of the the position of the partition gives no change in system energy but involves heat added so that

$$
T d \Delta S_{R J}=d \Delta U_{R J}+\Delta X_{R J} d x=0+\Delta X_{R J} d x
$$

Then using the Casimir force (25) associated with the Rayleigh-Jeans spectrum and like boundary conditions,

$$
d \Delta S_{R J}=\frac{X_{R J}}{T} d x=\frac{-k_{B}}{2}\left(\frac{1}{x}-\frac{1}{L-x}\right) d x
$$

and

$$
\left.\Delta S_{R J}(x, L, T)\right)=\frac{k_{B}}{2}\left|\ln \left(\frac{x}{L-x}\right)\right|
$$


We emphasize that the right-hand side of Eq.(31) is independent of temperature T. Thus due to the normal mode structure of the box, there is a temperature-independent change of entropy when the partition is moved. This seems reminiscent of the temperature-independent change associated with mixing entropy for ideal gas particles. This same result (31) can be obtained by summing the Rayleigh-Jeans entropy $\mathcal{S}(\omega / T)=-\ln (\omega / T)+1$ per normal mode over the normal modes of the box when using a cut-off function, subtracting, and allowing the cut-off to disappear.

The Casimir entropy change $\Delta S_{P}(x, L, T)=S_{P}(T, x)+S_{P}(T, L)-2 S_{P}(T, L / 2)$ associated with the Planck spectrum (and involving convergent sums) goes to the Rayleigh-Jeans limit (31) at high temperature for fixed values of $x$ and $L$. However, for fixed finite temperature $T$, the entropy change $\Delta S_{P}(x, L, T)$ for the Planck spectrum goes to a finite temperaturedependent limit as $x$ goes to 0 or $L$, as seen in Fig. 6 .

The Casimir entropy for unlike boundary conditions is seen in Fig. 7 to first increase from zero for increasing temperature and then to decrease back to zero for additional increase of temperature for fixed partition-coordinate $\mathrm{x}$. Thus in one dimension for unlike boundary conditions, there is no non-vanishing temperature-independent Casimir entropy in the hightemperature limit. We note that for the Rayleigh-Jeans spectrum, there is no Casimir energy $\Delta U_{R J}=0$ and, for unlike boundary conditions in one dimension, no Casimir force $\Delta \dot{X}_{R J}$. Accordingly, the first law of thermodynamics in (29) indicates that the Casimir entropy vanishes for the Rayleigh-Jeans spectrum and unlike boundary conditions.

The existence of temperature-independent Casimir entropy seems to depend sensitively on both the boundary conditions and dimension. The existence of a geometrical temperatureindependent Casimir entropy for like boundary conditions as given in (31) agrees with the result reported by Revzen, Opher, Opher and Mann for the case of two conducting parallel plates.[23] However, the absence of such an entropy for unlike boundary conditions is in contrast to the temperature-independent Casimir entropy reported by da Silva, Matos Neto, Pacido, Revzen, and Santana for the case two parallel plates, one of which is conducting and one of which is highly permeable.[17]

\section{CLOSING SUMMARY}

The the introduction of a partition into a wave system leads to changes in energies, forces, and thermodynamic variables associated with the wave boundary conditions at the partition and the walls. For electromagnetic waves, these Casimir forces are termed "dispersion forces" or "van der Waals forces." Although physicists usually think of van der Waals forces as attractive, the type of analysis introduced originally by Casimir suggests the possibility of repulsive forces. Several aspects of Casimir forces can be easily illustrated by a mathematical model involving waves in one space dimension. Depending upon the boundary conditions, a partition introduced into a one-dimensional box will experience attraction to or repulsion form the walls at zero temperature. The attractive force becomes increasingly attractive with increasing temperature whereas the repulsive force diminishes with increasing temperature. Also, there are changes in entropy associated with changes in position for the partition. For high temperatures, these entropy changes become temperature independent in the attractive case (rather like a wave form of mixing entropy) but vanish in the repulsive case. Finally, we note that Casimir energies are closely related to the thermodynamics of blackbody radiation, allowing a derivation of the Planck spectrum from a natural maximum-entropy principle. 


\section{ACKNOWLEDGEMENT:}

I would like to thank Professor Daniel C. Cole for sending me annotated copies of the work on repulsive Casimir forces by E. Buks and M. L. Roukes, and by O. Kenneth, I. Klich, A.. Mann, and M. Revzen . It was Professor Cole's communication which stimulated the present work. 


\section{Figure Captions}

Figure 1: Planck Spectrum Energy Per Normal Mode. The energy $\mathcal{U}_{P z p}(\omega, T)$ of the Planck spectrum with zero-point energy (13) is plotted vs mode angular frequency for three different temperatures. The sloping solid line for $T=0$ corresponds to the zero-point energy $\mathcal{U}_{z p}=(1 / 2) \hbar \omega$. The two horizontal lines correspond to the Rayleigh-Jeans spectra for the temperatures $T=1$ and $T=2$. The Planck expression for the energy per normal mode at $T>0$ starts with the equipartition value at low frequency and then goes smoothly to the zero-point curve at high frequency. The dashed curve for $T=1$ starts with the equipartition value $\mathcal{U}=1$ at low frequency, while the dotted curve for $T=2$ starts with the equipartition value $\mathcal{U}=2$. The Planck law interpolates smoothly between the Rayleigh-Jeans and zeropoint spectra.

Figure 2: Casimir Energy for Like Boundary Conditions. The Casimir energy $\Delta U_{P z p}(x, L, T)$, for the Planck spectrum with zero-point radiation in the case of like boundary conditions between walls and partition, is plotted as a function of the distance $x$ from the left-hand wall located at $x=0$ in a box of length $L=3$ for three different temperatures. The solid curve gives the negative Casimir energy (7) at temperature $T=0$. The dashed curve for $T=1$ gives Casimir energies close to zero near the center of the box before dropping down near the walls to join the curve for zero-point energy. The dotted curve at $T=3$ hugs the axis for a larger central interval. The Rayleigh-Jeans result (9), which holds for the high-temperature limit of the Planck spectrum, has zero Casimir energy for every value of $x$.

Figure 3: Casimir Energy for Unlike Boundary Conditions. The Casimir energy $\Delta U_{P z p}(x, L, T)$, for the Planck spectrum with zero-point radiation in the case of unlike boundary conditions between walls and partition, is plotted as a function of the distance $x$ from the left-hand wall located at $x=0$ in a box of length $L=3$ for three different temperatures. The solid curve gives the positive Casimir energy (8) at temperature $T=0$. The dashed curve for $T=1$ gives Casimir energies close to zero near the center of the box before rising near the walls to join the curve for zero-point energy. The dotted curve at $T=3$ hugs the axis for a larger central interval. The Rayleigh-Jeans result (9), which holds for the high-temperature limit of the Planck spectrum, has zero Casimir energy for all values of $x$.

Figure 4: Casimir Force for Like Boundary Conditions. The Casimir force $\Delta X_{P z p}(x, L, T)$, for the Planck spectrum with zero-point radiation in the case of like boundary conditions between walls and partition, is plotted as a function of the distance $x$ from the left-hand wall located at $x=0$ in a box of length $L=3$ for three different temperatures. The solid curve gives the Casimir force (15) which attracts the partition to the walls at temperature $T=0$. The dashed curve for $T=1$ shows an increased Casimir force which near the walls joins the curve for the zero-point Casimir force. The dotted curve at $T=3$ shows the continued increase with increasing temperature. The Rayleigh-Jeans result (25), which holds for the high-temperature limit of the Planck spectrum, has increasing Casimir force with increasing temperature.

Figure 5: Casimir Force for Unlike Boundary Conditions. The Casimir force $\Delta X_{P z p}(x, L, T)$, for the Planck spectrum with zero-point radiation in the case of unlike boundary conditions between walls and partition, is plotted as a function of the distance $x$ from the left-hand wall located at $x=0$ in a box of length $L=3$ for three different temperatures. The solid curve gives the Casimir force (16) which repels the partition from the walls at temperature $T=0$. The dashed curve for $T=1$ shows decreasing Casimir 
force in the central region of the box before leaving the axis near the walls to join the curve for the zero-point Casimir force. The dotted curve at $T=3$ shows the continued decrease with increasing temperature. The Rayleigh-Jeans result, which holds for the hightemperature limit of the Planck spectrum, has vanishing Casimir force at all temperatures for the one-dimensional case with unlike boundary conditions.

Figure 6: Casimir Entropy for Like Boundary Conditions. The Casimir entropy $\Delta S_{P}(x, L, T)$, for the Planck spectrum with zero-point radiation in the case of like boundary conditions between walls and partition, is plotted as a function of the distance $x$ from the left-hand wall located at $x=0$ in a box of length $L=3$ for several different temperatures. At zero temperature the Casimir entropy vanishes. The solid curve gives the Casimir entropy at $T=0.25$. The dashed curve for $T=0.5$ shows increasing Casimir entropy at this higher temperature away from the central region, which is continued in the dash-dot curve for $T=1$ and the dotted curve for $T=2$. In the central region, the Casimir entropy goes over to a temperature-independent result at high temperature. The Rayleigh-Jeans result, which holds for the high-temperature limit of the Planck spectrum, involves a temperatureindependent Casimir entropy (31) at all temperatures for a one-dimensional box with like boundary conditions.

Figure 7: Casimir Entropy for Unlike Boundary Conditions. The Casimir entropy $\Delta S_{P}(x, L, T)$, for the Planck spectrum with zero-point radiation in the case of unlike boundary conditions between walls and partition, is plotted as a function of the distance $x$ from the left-hand wall located at $x=0$ in a box of length $L=3$ for several different temperatures. At zero temperature the Casimir entropy vanishes. The solid curve gives the Casimir entropy at $T=0.1$. The dashed curve for $T=0.2$ shows increasing Casimir entropy at this higher temperature away from the central region. However, the dash-dot curve for $T=0.5$ shows a decrease of the Casimir entropy in the central region of the box and an increase near the walls. The dotted curve for $T=1$ shows the continued decrease in Casimir entropy in the central region. The Rayleigh-Jeans result, which holds for the high-temperature limit of the Planck spectrum, involves vanishing Casimir entropy at all temperatures for a one-dimensional box with unlike boundary conditions.

References

[1] See S. K. Lamoreau, "Resource Letter CF-1: Casimir Force," Am. J. Phys. 67, 850-861 (1999); M. Bordag, U. Mohideen, and V. M. Mostepanenko, "New developments in the Casimir effect," Physics Report 353, 1-205 (2001).

[2] H. B. G. Casimir, "On the attraction between two perfectly conducting plates," Proc. Kon. Ned. Akad. Wetenschap. 51, 793-795 (1948).

[3] V. M. Mostepanenko and N. N. Trunov, The Casimir Effect and its Applications, (Oxford U. Press, Oxford 1997); P. W. Milonni, The Quantum Vacuum: An Introduction to Quantum Electrodynamics, (Academic Press, New York 1994).

[4] A. Larraza, "A demonstration apparatus for an acoustic analog to the Casimir effect," Am. J. Phys. 67, 1028-1030 (1999).

[5] S. L. Boersma, "A maritime analogy of the Casimir effect," Am. J. Phys. 64, 539-541 (1996).

[6] D. Bishop, P. Gammel, and C. R. Giles, "The little machines that are making it big," Physics Today, pp. 38-44 October 2001. 
[7] M. J. Sparnaay, "Measurement of the attractive forces between flat plates," Physica 24, 751764 (1958); S. K. Lamoreaux, "Demonstration of the Casimir force in the 0.6 to $6 \mu \mathrm{m}$ range," Phys. Rev. Lett. 78, 5-8 (1997), 81, 5475-5476 (1998); U. Mohideen, "Precision measurement of the Casimir force from 0.1 to $0.9 \mu \mathrm{m}$," Phys. Rev. Lett. 81, 4549-4552 (1998); and H. B. Chan, V. A. Aksyuk, R. N. Kleiman, D. J. Bishop, and F. Capasso, "Quantum mechanical actuation of microelectromechanical systems by the Casimir force," Science 291, 1941-1944 (2001); G. Bressi, G. Carugno, R. Onofrio, and G. Ruoso, "Measurement of the Casimir force between parallel metallic surfaces," Phys. Rev. Lett. 88, 041804(4) (2002).

[8] E. Buks and M. L. Roukes, "The Casimir force changes sign," Nature 419, 119-120 (2002); O. Kenneth, I. Klich, A. Mann, and M. Revzen, "Repulsive Casimir forces," Phys. Rev. Letters 89, 033001(4) (2002).

[9] Examples of non-thermal spectra are found in the acoustic analogue of the Casimir effect in ref. 4 and the one-dimensional effect discussed by D. J. Griffiths and E. Ho, "Classical Casimir effect for beads on a string," Am. J. Phys. 69, 1173-1176 (2001).

[10] Discussions of the Wien displacement law are given by, for example, M. Planck, The Theory of Heat Radiation (Dover, New York 1959), 72-83, or F. K. Richtmyer, E. H. Kennard, and T. Lauritsen, Introduction to Modern Physics (McGraw-Hill, New York 1955), pp. 113-118, or B. H. Lavenda, Statistical Physics: a Probabilistic Approach (Wiley, New York 1991), pp. 67-69. A very different derivation is presented by T. H. Boyer, "Thermodynamics of the harmonic oscillator: Wien's displacement law and the Planck spectrum," submitted for publication. (physics/0210128) The notation of the present work is that of the last article.

[11] Here $\mathcal{U}$ is written in terms of the thermodynamic potential $\phi(\omega / T)$ used by C. Garrod, Statistical Mechanics and Thermodynamics (Oxford, New York 1995), p. 128.

[12] See for example, G. H. Hardy, Divergent Series (Oxford University Press, London, 1956).

[13] See, for example, T. H. Boyer, "Van der Waals forces and zero-point energy for dielectric and permeable materials," Phys. Rev. A 9, 2078-2084 (1974).

[14] See, for example, F. S. Crawford, Waves (McGraw-Hill, New York 1968), p. 56 and p. 68.

[15] See ref. 13, and also V. Hushwater, "Repulsive Casimir force as a result of vacuum radiation pressure," Am. J. Phys. 65, 381-384 (1997); J. C. da Silva, A. Matos Neto, H. Q. Placido, M. Revzen, and A. E. Santana, " Casimir effect for conducting and permeable plates at finite temperature," Physica A 292, 411-421 (2001). A repulsive aspect of van der Waals forces was suggested in the calculation for a spherical geometry by T. H. Boyer, "Quantum electromagnetic zero-point energy of a conducting spherical shell and the Casimir model for a charged particle," Phys. Rev. 174, 1764-1776 (1968).

[16] M. Revzen, R. Opher, M. Opher, and A. Mann, "Kirchhoff's theorem and the Casimir effect," Europhys. Lett. 38, 245-248 (1997).

[17] J. C. da Silva, A. Matos Neto, H. Q. Placido, M. Revzen, and A. E. Santana, " Casimir effect for conducting and permeable plates at finite temperature," Physica A 292, 411-421 (2001).

[18] T. H. Boyer, "Derivation of the blackbody radiation spectrum from a natural maximumentropy principle involving Casimir energies and zero-point radiation," submitted for publication. (physics/0210129)

[19] This same axis-hugging behavior for the Planck spectrum can be seen in the three-dimensional electromagnetic case for conducting boundary conditions in T. H. Boyer's "Some Aspects of Quantum Electromagnetic Zero-Point Energy and Retarded Dispersion Forces," Harvard doctoral thesis 1968 (unpublished), Fig. 4.

[20] See the "Concluding Remarks" of J. C. da Silva, A. Matos Neto, H. Q. Placido, M. Revzen, 
and A. E. Santana, in ref. 17.

[21] F. Ferrer and J. A. Grifols, "Casimir-Polder forces in the presence of the cosmic photon heat bath," Phys. Letters B 511, 319-320 (2001).

[22] The long-range interaction between an electrically polarizable particle and a magnetically polarizable one was first given by G. Feinberg and J. Sucher, J. Chem. Phys. 46, 3333 (1968). These interactions can be regarded as involving shifts of electromagnetic energy in the radiation field such as are involved in Casimir forces; see T. H. Boyer, "Recalculations of long-range van der Waals potentials," Phys. Rev. 180, 19-24 (1969).

[23] M. Revzen, R. Opher, M. Opher, and A. Mann, "Casimir's entropy," J. Phys. A: Math. Gen. 30, 7783-7789 (1997). 


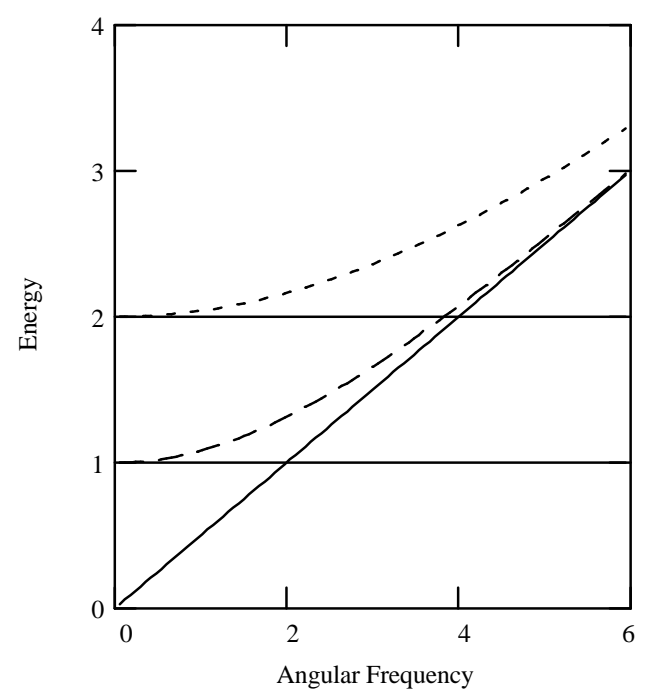

Fig. 1: Planck Spectrum Energy

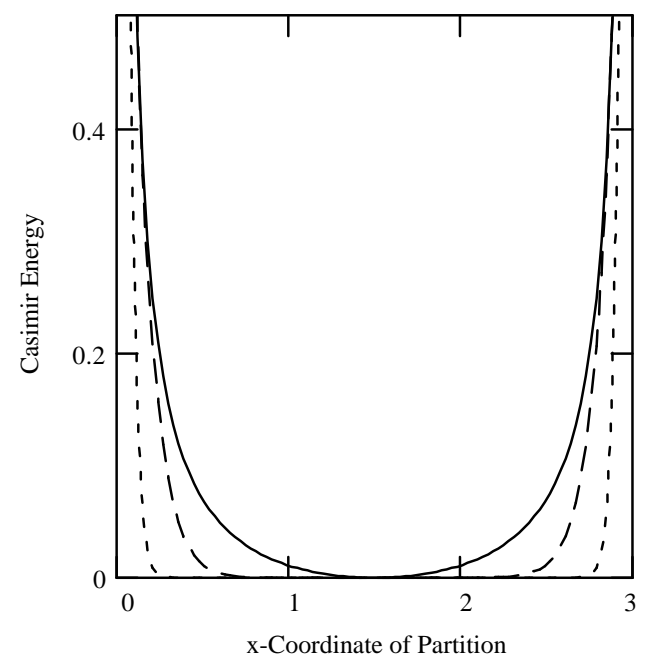

Fig. 3: Casimir Energy for Unlike BC

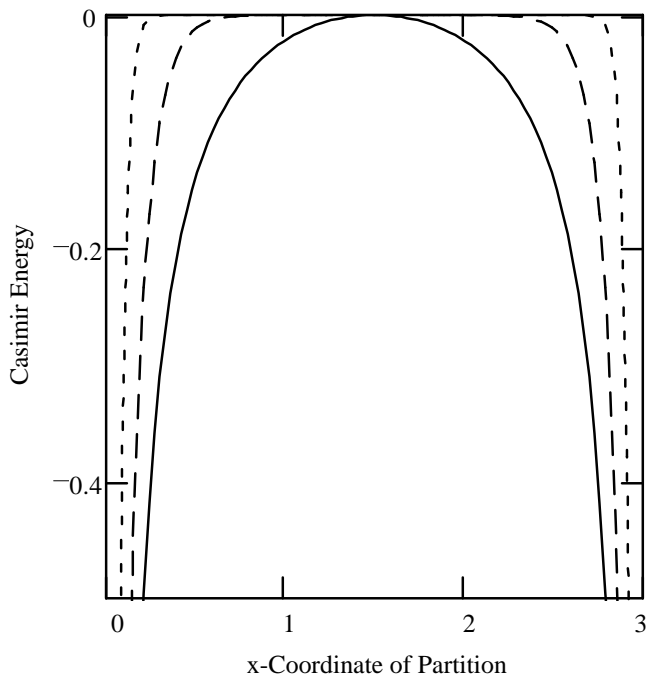

Fig. 2: Casimir Energy for Like BC

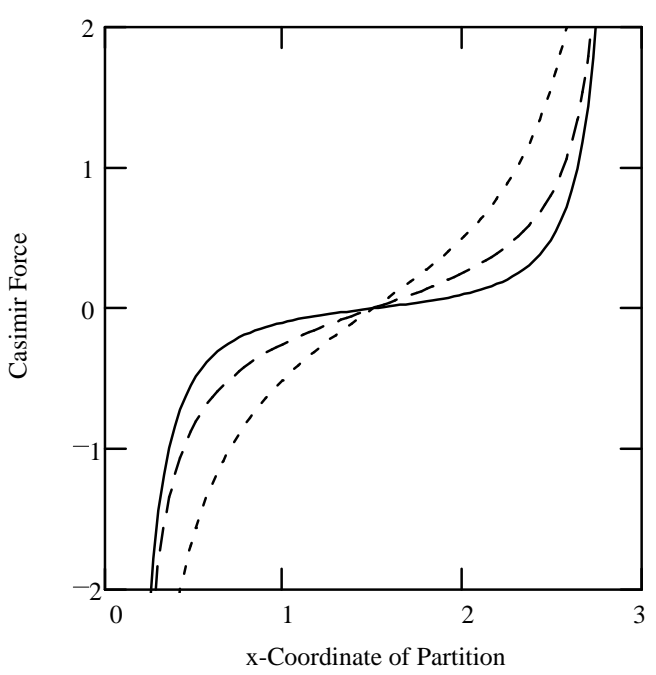

Fig. 4: Casimir Force for Like BC 


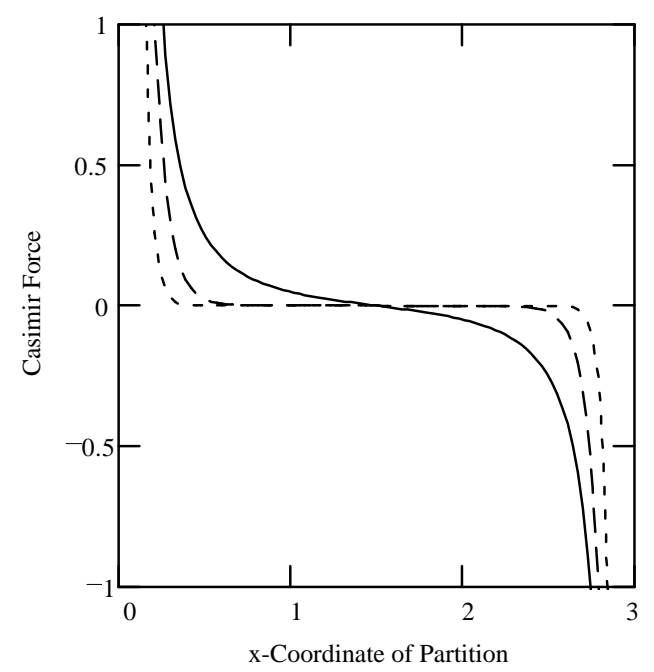

Fig. 5: Casimir Force for Unlike BC

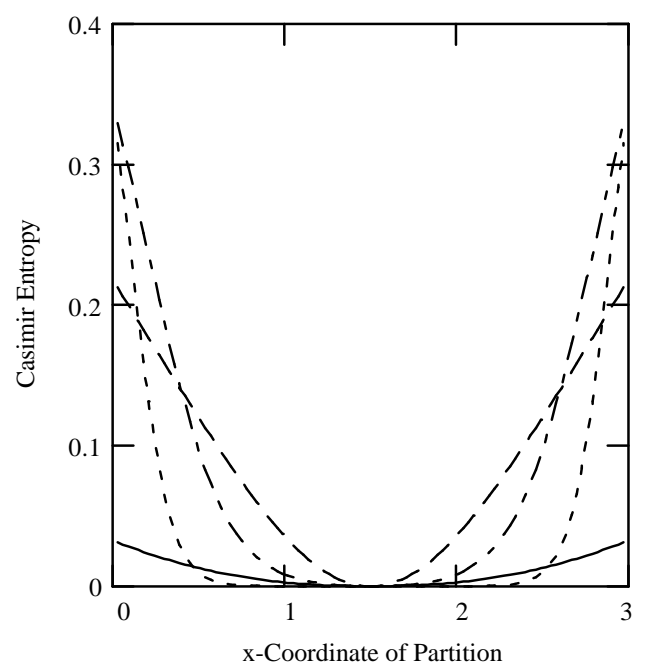

Fig. 7: Casimir Entropy for Unlike BC

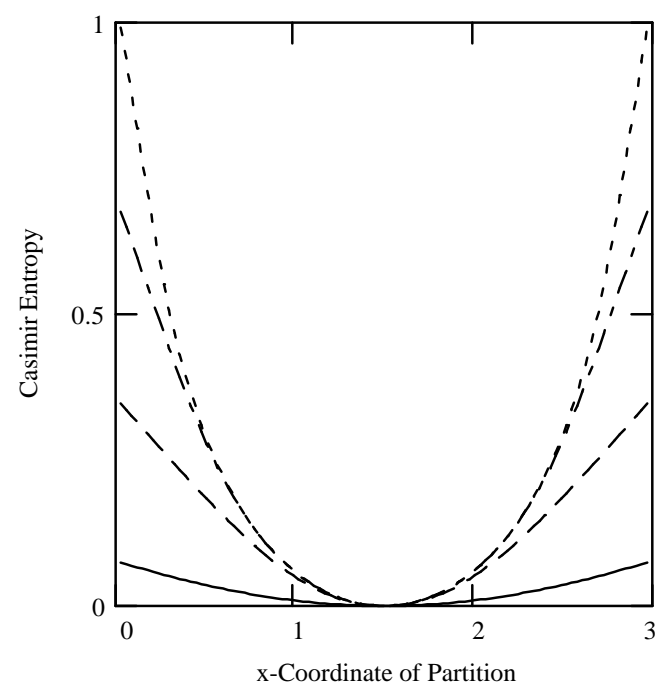

Fig. 6: Casimir Entropy for Like BC 